\title{
Characterization of Biological Secretions Binding to Graphene Oxide in Water and the Specific Toxicological Mechanisms
}

Li Mu${ }^{\dagger}$, Yue $\mathrm{Gao}^{\ddagger}$, Xiangang $\mathrm{Hu}^{\ddagger}$

${ }^{\dagger}$ Institute of Agro-environmental Protection, Ministry of Agriculture, Tianjin 300191, China

${ }^{\ddagger}$ Key Laboratory of Pollution Processes and Environmental Criteria (Ministry of

Education), Tianjin Key Laboratory of Environmental Remediation and Pollution

Control, College of Environmental Science and Engineering, Nankai University,

Tianjin 300071, China.

Number of Pages: 9

Number of Figures: 9

Number of Tables: 1

\footnotetext{
* Corresponding author at: College of Environmental Science and Engineering, Nankai University, Tianjin 300071, China. E-mail address: huxiangang@nankai.edu.cn (X.G. Hu)
} 


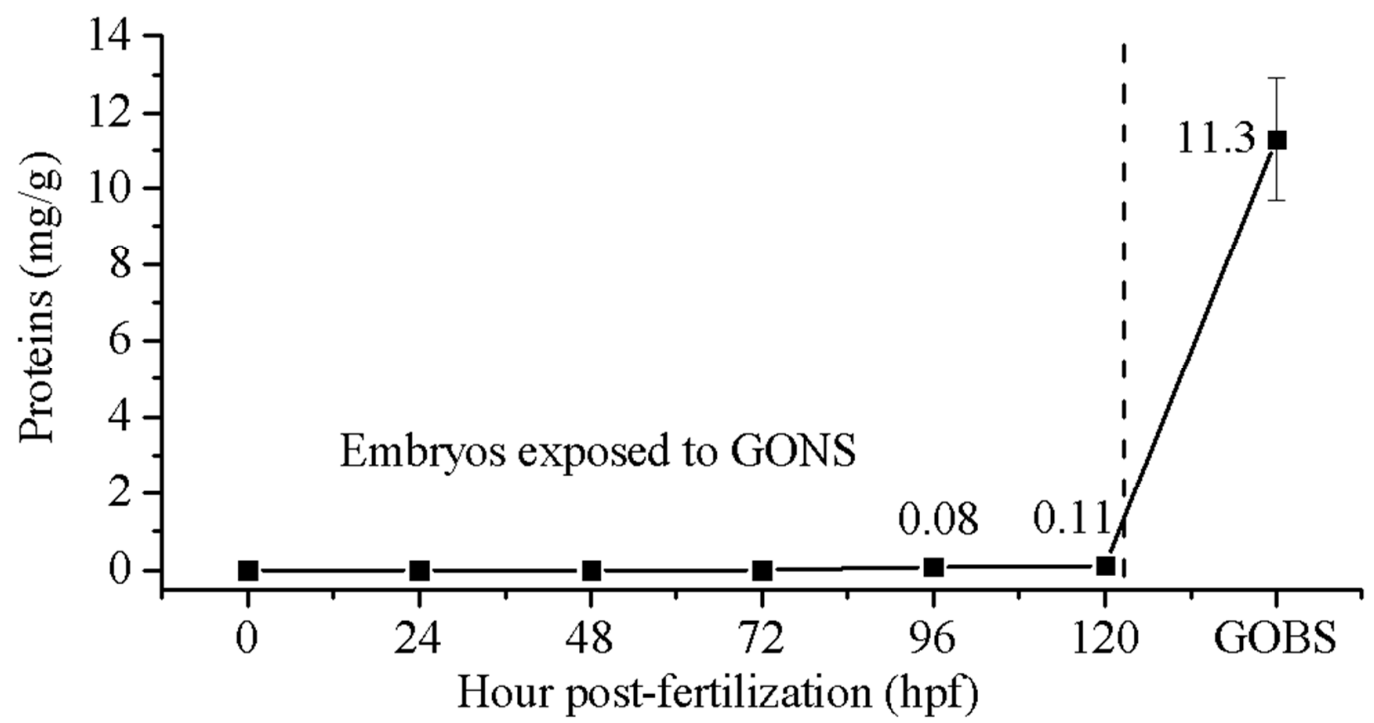

Figure S1. Proteins on nanomaterials. The left of dotted line is the proteins on graphene oxide nanosheets (GONS) during embryo exposure experiment. During the exposure, embryo culture medium is replaced every $24 \mathrm{~h}$ until $120 \mathrm{hpf}$. The right of dotted line is the proteins on graphene oxide nanosheets with biological secretions (GOBS). GOBS are GONS reacting with the secretions from adult zebrafish for $72 \mathrm{~h}$. 


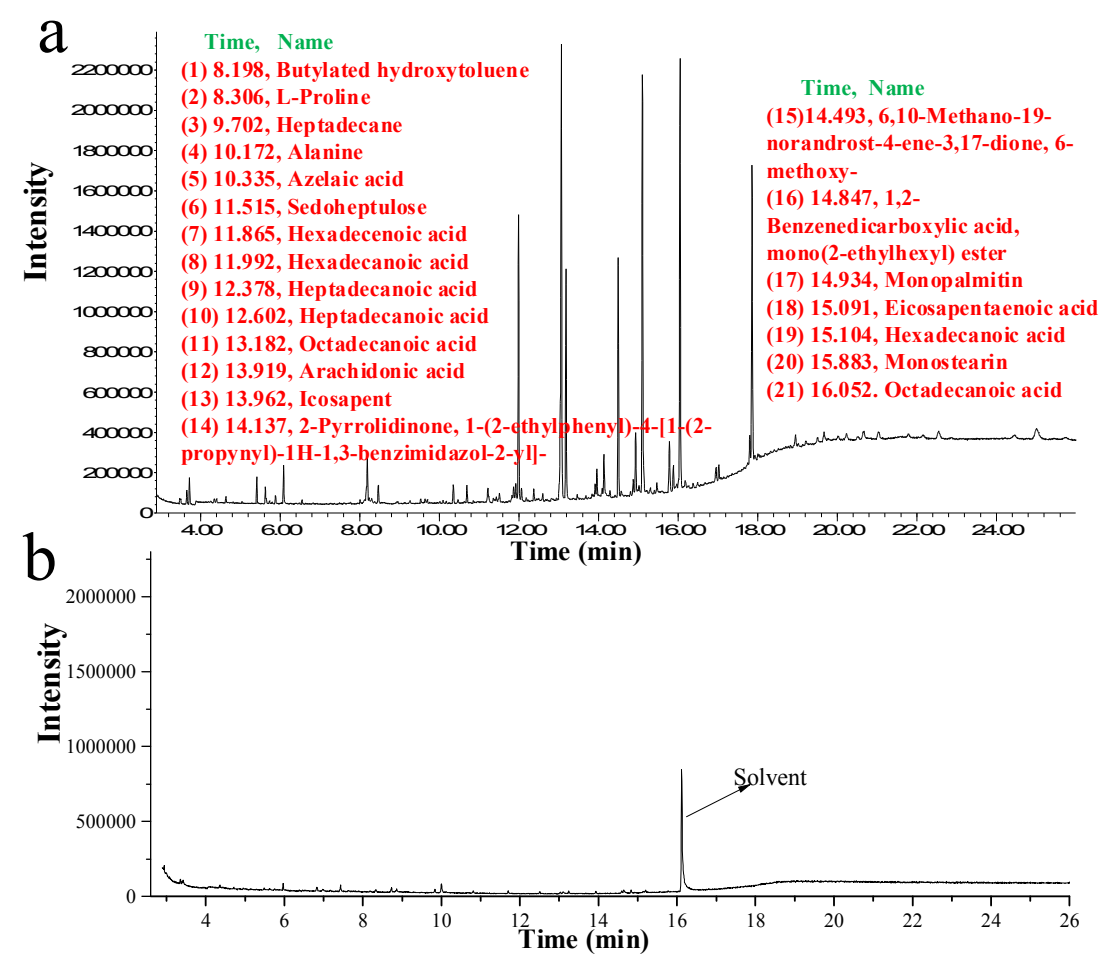

Figure S2. Small organic molecules on nanomaterials detected using gas

chromatography combined with quadrupole mass spectrometry. a, Small organic molecules on graphene oxide nanosheets with biological secretions. b, Small organic molecules on graphene oxide nanosheets. 

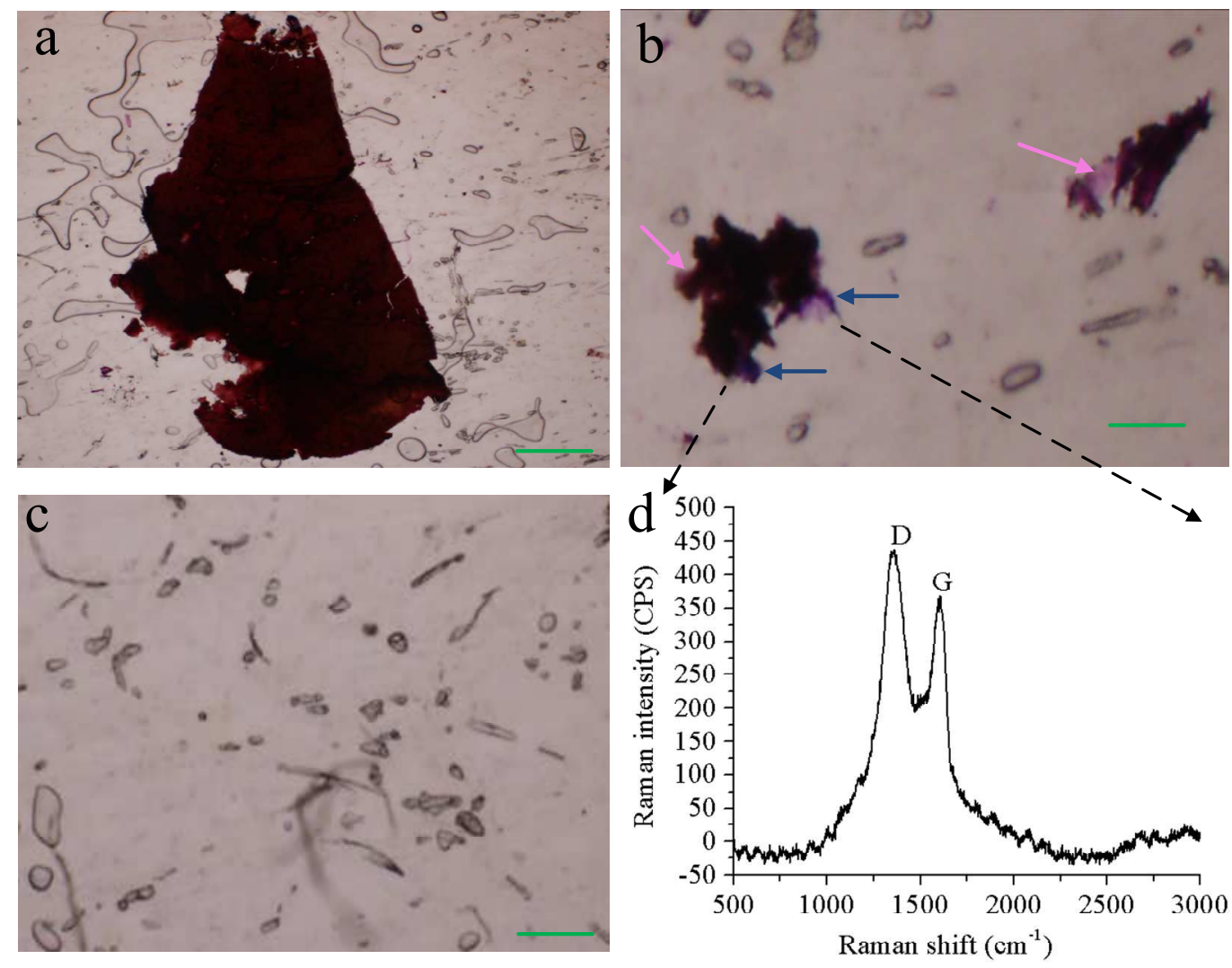

Figure S3. Alcian blue and periodic acid-Schiff staining of powders of GONS and GOBS. a, Alcian blue and periodic acid-Schiff staining of powders of GONS. b, Alcian blue and periodic acid-Schiff staining of powders of GOBS. c, Glue (acrylate polymer) on glass slide; d, Raman spectrum of powders of GOBS. Blue and pink arrows denote acid and neutral mucopolysaccharides on GOBS, respectively. Scale bars, $0.5 \mathrm{~mm}$.

Table S1. Concentrations of secretions on nanomaterials

\begin{tabular}{lll}
\hline & GONS & GOBS \\
\hline Proteins & Not found & $11.3 \pm 1.6 \mathrm{mg} / \mathrm{g}$ \\
DNA & Not found & $8.3 \pm 1.4 \mathrm{mg} / \mathrm{kg}$ \\
Others $^{*}$ & Not found & $23.4 \pm 0.8 \mathrm{mg} / \mathrm{g}$ \\
\hline
\end{tabular}

"Others" = total secretions on nanomaterials - proteins $-\mathrm{DNA}$; total secretions on nanomaterials = GONS Mass at $700^{\circ} \mathrm{C}-$ GOBS Mass at $700^{\circ} \mathrm{C}=43.0 \mathrm{mg} / \mathrm{g}$. 

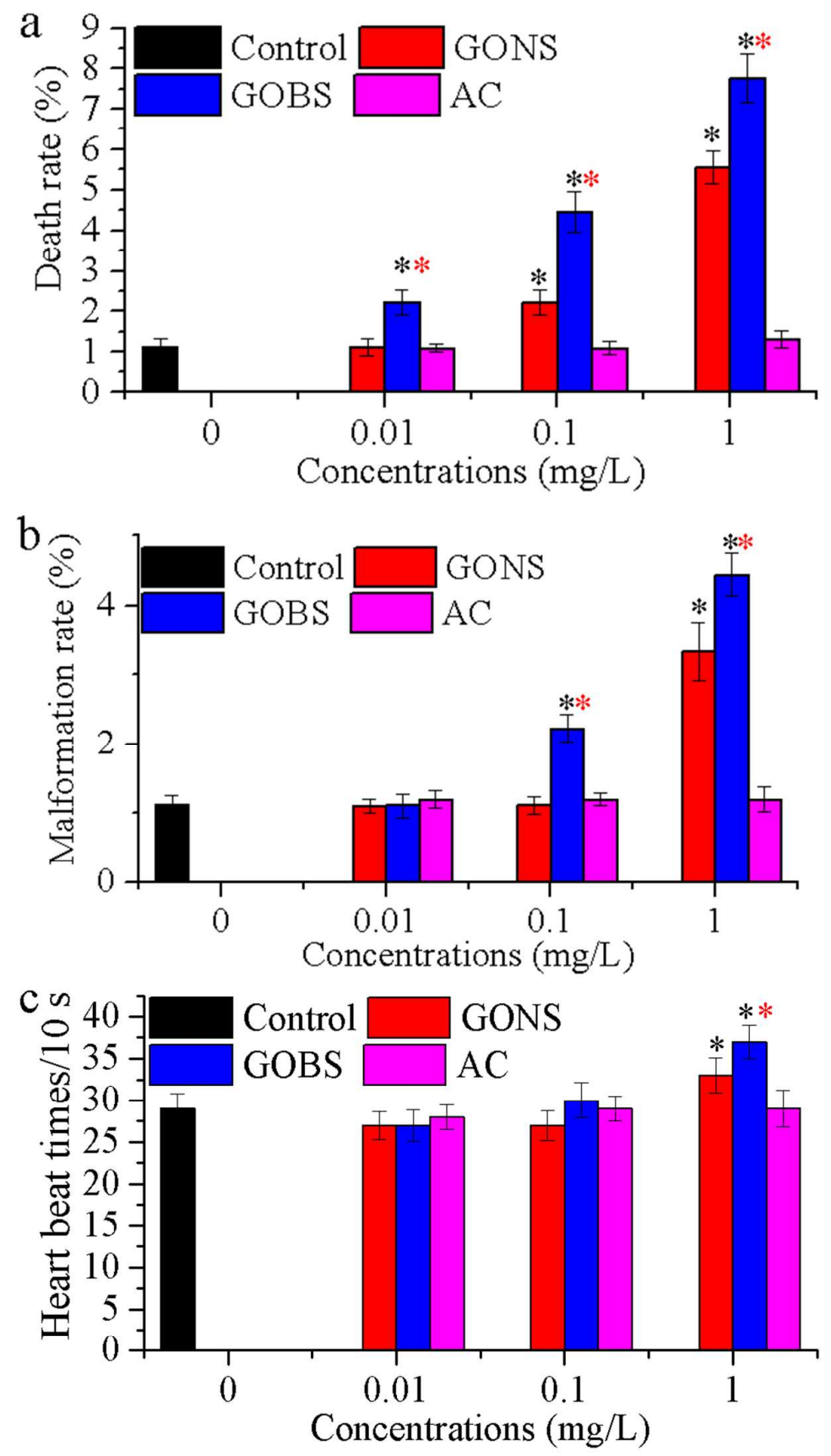

Figure S4. Effects of GONS and GOBS on the development of zebrafish embryos at 120 hpf. a, Percentage of death; b, percentage of malformation; c, heartbeat times. The black and red asterisks represent significance at $P<0.05$ compared to the control and GONS, respectively. Each experiment was conducted in triplicate, with 20 embryos per replicate. GONS, graphene oxide nanosheets; GOBS, graphene oxide nanosheets with biological secretions; AC, activated carbon. 

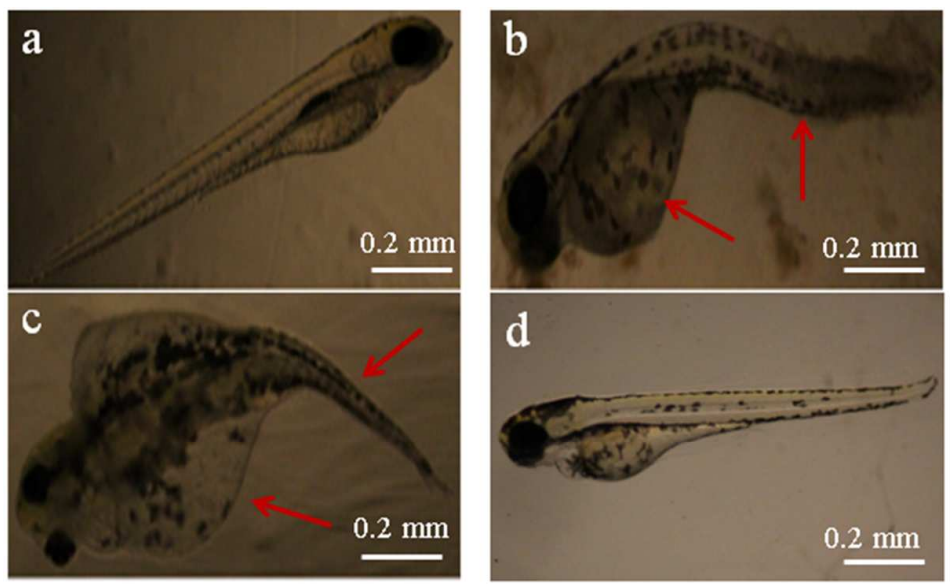

Figure S5. Microscopic imaging of embryonic development after exposure to GONS or GOBS. a, Control; b, GONS; c, GOBS; d, AC. The red arrows indicate tail flexure and pericardial edema. GONS, graphene oxide nanosheets; GOBS, graphene oxide nanosheets with biological secretions; AC, activated carbon. 

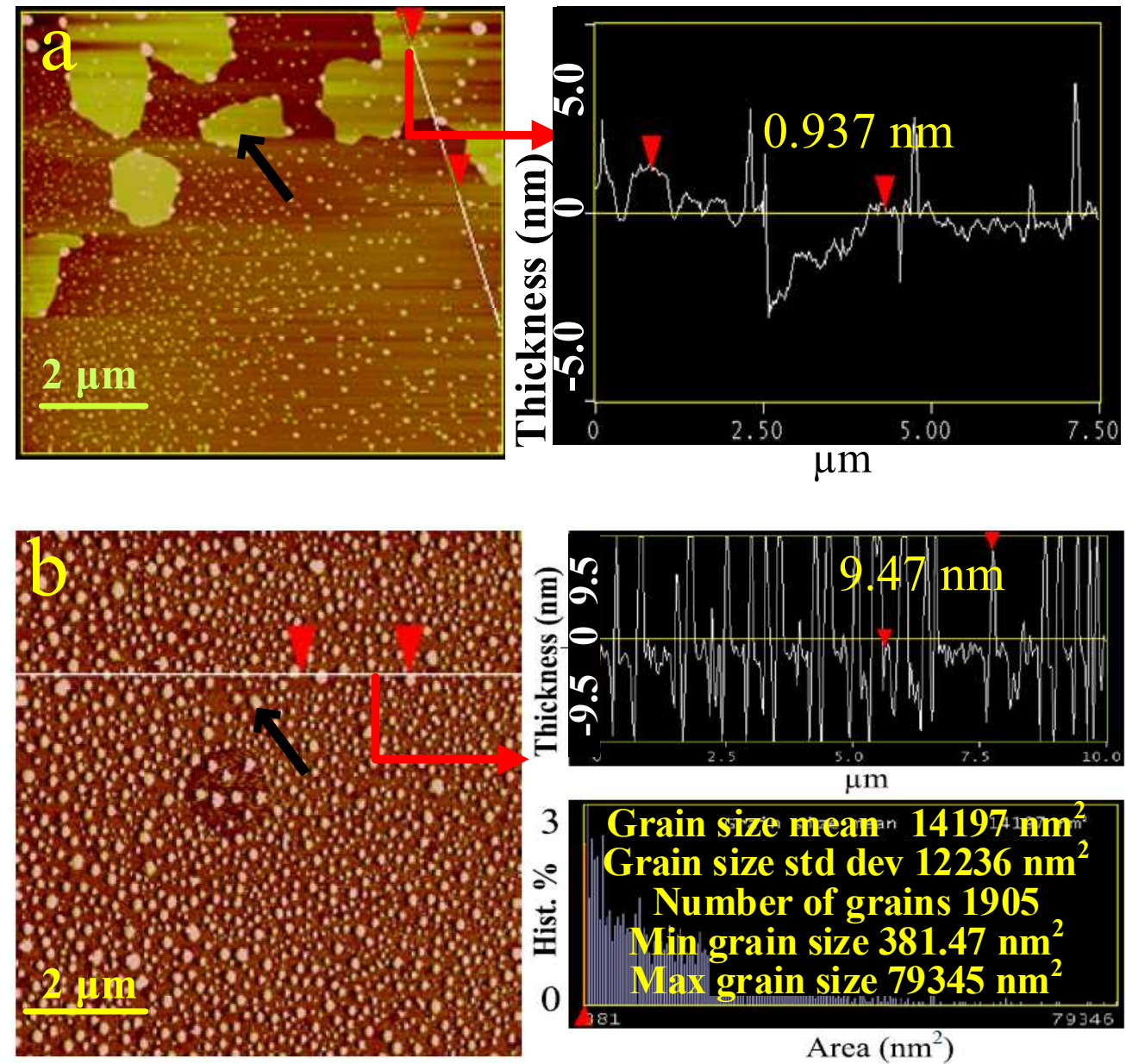

Figure S6. Atomic force microscopic images of GONS (a) and GOBS (b). The black arrows denote nanomaterials. GONS, graphene oxide nanosheets; GOBS, graphene oxide nanosheets with biological secretions. 

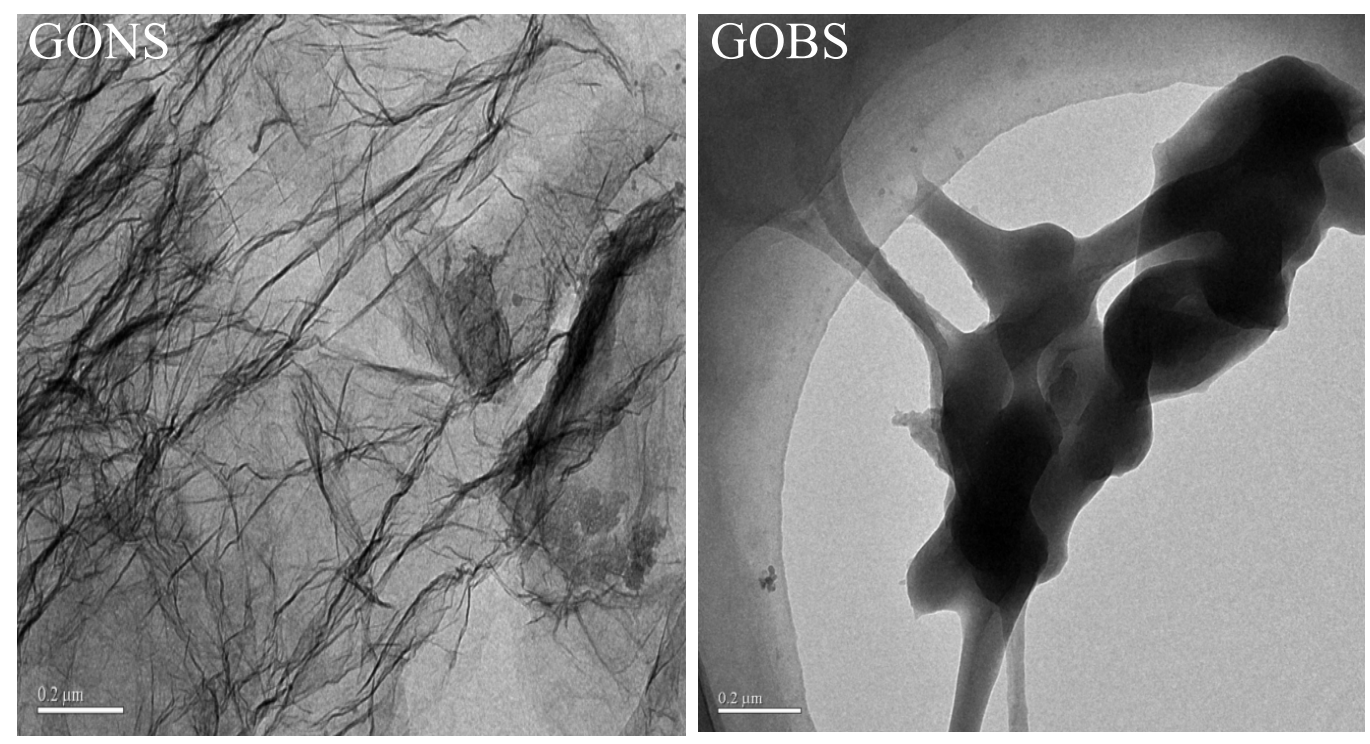

Figure S7. Field-emission transmission electron microscopy of graphene oxide nanosheets (GONS, Figure S7a) and graphene oxide nanosheets with biological secretions (GOBS, Figure S7a). The scale bars are $0.2 \mu \mathrm{m}$.

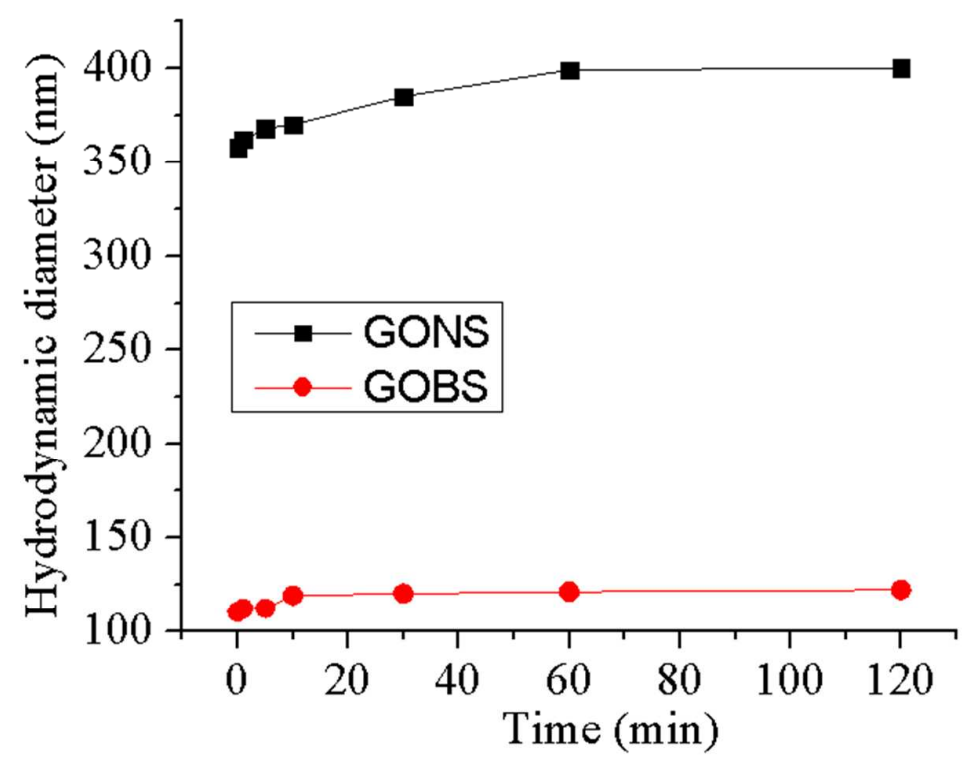

Figure S8. Alterations in the hydrodynamic diameters of GONS and GOBS with time. The tested concentration of nanomaterials is $1 \mathrm{mg} / \mathrm{L}$. GONS, graphene oxide nanosheets; GOBS, graphene oxide nanosheets with biological secretions. 


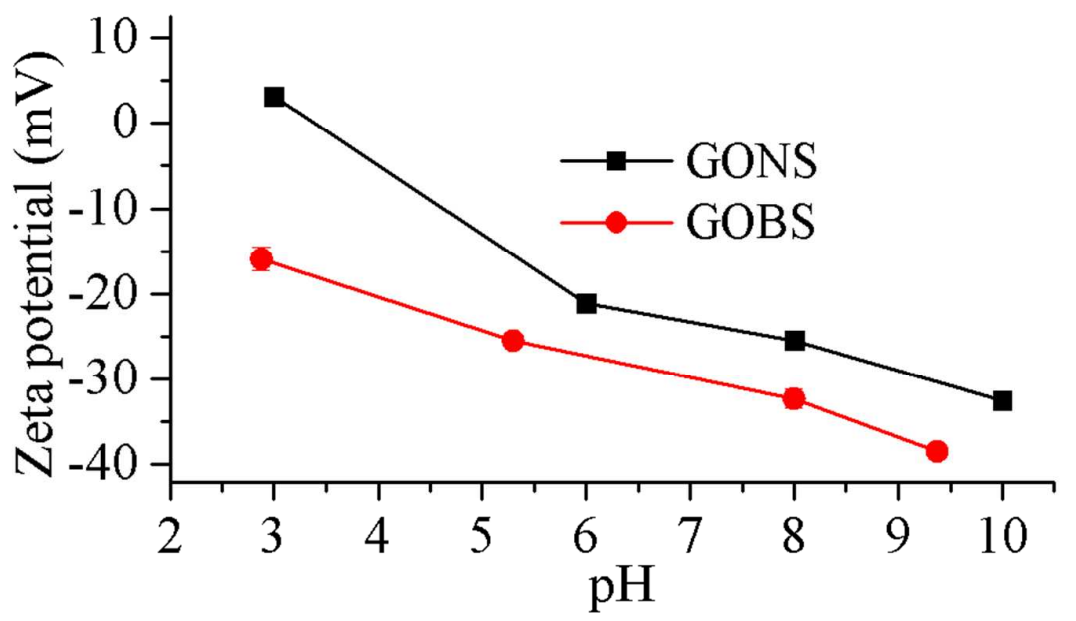

Figure S9. Zeta potential profiles of GONS and GOBS. GONS, graphene oxide nanosheets; GOBS, graphene oxide nanosheets with biological secretions. 